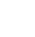

\title{
End-of-life psychodrama: Influencing nursing students' communication skills, attitudes, emotional intelligence and self-reflection
}

\author{
Audrey Marie Beauvais, ${ }^{1} \odot$ Azize Atli Özbaş, $^{2} \odot$ Kathleen Wheeler $^{1}$ \\ 'Egan School of Nursing and Health Studies, Fairfield University, Fairfield, CT United States \\ ${ }^{2}$ Department of Psychiatric Nursing, Hacettepe University Faculty of Nursing, Ankara, Turkey
}

\begin{abstract}
Objectives: This study aimed to determine if nursing students' communication skills, attitudes towards caring of the dying patients, emotional intelligence, and reflection change after a psychodrama group intervention.

Methods: A pre/posttest design was utilized with a psychodrama intervention group and a control group during a Mental Health Nursing course. The study was set in Fairfield University (USA) with approximately 390 traditional undergraduate nursing students. A convenience sample of eighty-four nursing students was invited to participate in the study. Thirty-eight of those students participated in the intervention group and 41 participated in the control group. All participants in the control and intervention groups were asked to complete the demographic information, process recordings, Frommelt Attitude Toward Care of the Dying Scale, Mayer-Salovey-Caruso Emotional Intelligence Test, and Self Reflection and Insight Scale at the beginning and end of the psychodrama intervention.

Results: There was a statistically significant difference in communication skills, attitudes towards the care of the dying, and self-reflection between the intervention and control groups. There was no statistical difference in total and branch emotional intelligence between the intervention group and control groups.

Conclusion: The study highlighted the value of psychodrama as a strategy that can enhance nursing students' communication skills, attitudes towards dying patients, and reflection. Such an intervention has the potential to ultimately improve the quality of care for end-of-life patients and their families.
\end{abstract}

Keywords: Attitude; emotional intelligence; nursing; psychodrama; student; terminal care.

\section{What is known on this subject?}

- Nursing students can benefit from psychodrama education to improve end-of-life communication skills. Nursing students can benefit from psychodrama education to improve their attitudes towards the care of the dying.

What is the contribution of this paper?

- Although psychodrama might not influence overall emotional intelligence, it may enhance emotional intelligence skills.

What is its contribution to the practice?

- Nursing students can benefit from psychodrama education to improve their self-reflection and insight.
Effective communication skills are fundamental to all Egood nursing care. Perhaps nowhere is this more important than in working with patients who are at the end-oflife. However, ineffective communication skills have continued to be identified as problematic for nurses working with end-of-life patients and many nurses receive insufficient or inadequate training in this area. ${ }^{[1]}$ There is increasing recognition of the importance of communication skills training for nurses in this area and a lack of empirical evidence about how to enhance communication skills for end-of-life care. ${ }^{[2,3]}$

Address for correspondence: Audrey Marie Beauvais, 1073 North Benson Road 06890 Fairfield - United States Phone: 203-254-4000 E-mail: abeauvais@fairfield.edu ORCID: 0000-0001-8388-9981

Submitted Date: October 17, 2017 Accepted Date: March 11, 2019 Available Online Date: July 02, 2019

${ }^{\circ}$ Copyright 2019 by Journal of Psychiatric Nursing - Available online at www.phdergi.org 
Reviews in the medical education literature have provided evidence that those programs that incorporate interactive and multidimensional methods of teaching are often more successful in helping individuals to acquire communication skills in comparison to more didactic methods of teaching. ${ }^{[4]}$ Role-play is a commonly used technique to teach communication skills. ${ }^{[4]}$ However, role-play has been criticized because students are often reluctant to participate and are anxious in such situations. ${ }^{[4]}$ This study combines role-play techniques with active teaching methods using psychodrama techniques such as warm-ups, role reversal, mirroring and doubling in the psychodrama group sessions. Psychodrama provides a supportive context for the role play of communication techniques.

Psychodrama is a multidimensional method that facilitates internal changes, resulting in greater insight, personal growth, enhanced emotional intelligence and integration on cognitive, affective, and behavioral levels..$^{[5]}$ In psychodrama, the individual sees him/herself from outside in stages, progresses in self-recognition, and seizes the opportunity to re-evaluate the condition when $s /$ he sees the same stage from different angles or from the viewpoint of others. ${ }^{[5]}$ The individual is able to experience non-conventional solutions in the safe environment provided by the psychodramatist. ${ }^{[5]}$ Psychodrama employs guided dramatic action to examine problems or issues raised by an individual by using experiential methods, sociometry, role theory, and group dynamics. ${ }^{[5]}$

Psychodrama allows student nurses to increase self-awareness, develop communication skills, enhance their ability to cope with communication problems, and establish positive interpersonal relations. ${ }^{[6]}$ Although communication skills are taught in baccalaureate nursing programs, good communication involves more than rote mastery of skills. Underlying sensitive appropriate communication skills are the student's attitudes towards the patient and the student's ability to evaluate and clarify one's own thoughts, feelings and behaviors. These metacognitive factors are central to purposeful, therapeutic communication. This process of metacognition or reflection has been identified in the nursing literature as crucial to integrate into nursing education. ${ }^{[6]}$ Since psychodrama is designed to promote reflection, it is posited that psychodrama will enhance the student's ability to evaluate how and when to communicate appropriately with those at the end-of-life. It is only through internal changes that attitudes towards the care for those at the end-of-life change and consequently enduring appropriate communication skills are established.

A closely aligned concept that is important for internal change and reflection is that of emotional intelligence. Although several definitions of emotional intelligence can be found in the literature, this study theoretically defines emotional intelligence as the ability to perceive emotions, to integrate emotions to assist thought, to understand emotions and emotional knowledge, and to reflectively regulate and manage emotions so as to promote emotional and intellectual growth.
[7] There are many studies that have demonstrated the relationship between emotional intelligence and communication skills. ${ }^{[8-11]}$ Nursing academia has also found that emotional intelligence is linked to positive student outcomes such as nursing performance, academic success, resilience, spirituality, and empowerment. ${ }^{[12-14]}$ Given the relevance and importance of emotional intelligence and reflection to good communication skills in nursing education, this study will evaluate the effects of a psychodrama group intervention on these qualities.

Psychodrama has been used with nurses and patients with positive outcomes. For example, psychodrama helped to improve empowerment and decrease burnout in oncology nurses. ${ }^{[15]} \mathrm{A}$ qualitative study by Giusti et al. ${ }^{[16]}$ explored the lived experiences of patients adjusting to cancer via participation in a psychodrama intervention. The psychodrama intervention helped patients to achieve insights. Several recent studies have explored the use of psychodrama with nursing students. For example, Oflaz, et al. ${ }^{[6]}$ noted improved nursing student self-awareness after a psychodrama intervention. Students expressed that they could recognize fundamental emotions produced by the intervention which in turn may help to examine issues in caring experiences. In addition, Ozcan et al. ${ }^{[17]}$ demonstrated that psychodrama improved empathy (a concept closely related to emotional intelligence) in nursing students. These researchers recommended that other communication skills should be assessed after psychodrama with a control group. No studies could be found that explored if nursing students' communication skills, attitudes towards dying patients, emotional intelligence, and self-reflection and insight change after a psychodrama intervention.

The theoretical foundation for this study is based on Hildegard Peplau's theory of interpersonal relations. Her theory of interpersonal relations emphasizes the reciprocity between nurses and patients. The theory depicts nursing as an interactive and collaborative process between the nurse and the patient. Inherent to the theory is the idea that the nurse and patient respect each other as individuals and both learn and grow as a result of the interaction. Peplau's model is consistent with other communication models that include goals, plans, and action theories, sociolinguistic theory and Leventhal's common-sense model. ${ }^{[18]}$ These frameworks further explain why communication education optimizes patient outcomes. Integration of these models includes assisting the nurse to sensitively respond to the patient's need for explanations and recognizing the dissonance between their understanding and the medical explanation for treatment. This involves creating collaborative goals with the patient and the family. Central to good communication is recognizing the needs of each person. Foundational to the ability to attune to another's needs is the nurse's ability to perceive emotions in others, to integrate emotions to assist thought, and to reflectively regulate and manage one's own emotions.

Since psychodrama promotes reflection and self-awareness, it is hypothesized that psychodrama will enhance the student's 
ability to evaluate how and when to communicate appropriately with those at the end-of-life through their own internal transformation. Hence, the purpose of this study was to determine if nursing students' communication skills, attitudes towards dying patients, emotional intelligence, and reflection change after a psychodrama group intervention.

\section{Materials and Method}

\section{Participants}

The study was a pre/posttest design to examine if 6 weeks of psychodrama groups held as part of the student's clinical experience improves communication skills, attitudes toward care of those at the end-of-life, emotional intelligence, and self-reflection. The study took place in Fairfield University in the United States of America that has approximately 4,000 undergraduate students in attendance. The undergraduate nursing program has approximately 390 students. A convenience sample of 84 traditional undergraduate nursing students consisting of the class of 2017 was used. Forty-two students enrolled in the spring 2015 Mental Health Nursing Class were invited to participate in the intervention group. The other forty-two students enrolled in the fall 2015 Mental Health Nursing Class were invited to participate in the control group and did not receive psychodrama. The inclusion criterion for the study was current enrollment as a nursing student in the class of 2017 and volunteering to participate to the study. Both part-time and full-time students were eligible. Subjects under the age of 18 were excluded from the study as they are considered minors.

\section{Instruments}

Communication skills were measured by process recordings that were completed by students on the first and last day of clinical. The process recording is a verbatim, written account of an interaction between a client and nursing student. Through the reconstruction of the interaction, the student is provided with an opportunity to retrospectively examine and analyze his/her facilitative communication skills and therapeutic use of self and the client's contribution to the interaction. The student analyzes what is said (the content) and the flow of the interaction (the process). This analysis can help the student to increase awareness of his/her own feelings, values, attitudes, expectations, assumptions and beliefs, and how they influenced the interaction with the client. This analysis also helps the student to distinguish between his/her own thoughts and feelings, and to gain insight about how each influences his/her perception of the client, the clients' situation, and the effectiveness of the client's coping mechanisms. The process recordings were evaluated with a standardized grading rubric and a numerical grade was assigned out of 100 points. Various components of the process are assigned points such as the student's ability to separate out their thoughts from their feelings; an analysis of discrepancies between verbal and nonverbal behavior; labeling barriers to facilitating the relationship etc. The same clinical faculty graded the process recordings in the intervention and control group. Clinical faculty were trained how to use the process recordings by the coordinator of the Mental Health course who reviewed their ratings to ensure inter rater reliability.

Attitudes towards the care of the dying was measured by the Frommelt Attitude Toward Care of the Dying (FATCOD) Scale, Form $\mathrm{B}^{[19]}$ which is a 30 -item tool using a five-point Likert scale to indicate respondents' attitudes toward caring for dying patients. The instrument consists of an equal number of positively and negatively worded statements with response options of strongly disagree, disagree, uncertain, agree, and strongly agree. Positive items are scored one (strongly disagree) to five (strongly agree). Scores are reversed for negative items. Possible scores can range from 30-150. A higher score indicates a more positive attitude toward caring for this patient population. Reliability of the FATCOD has been established multiple times ${ }^{\left[{ }^{[9]}\right.}$ Frommelt used a test-retest method with a sample of 18 oncology nurses wherein nurses responded to the instrument and repeated it again three weeks later. The computed Pearson coefficient was found to be 0.94 . A sample of 30 nurses (mixed oncology and surgical) later was used to strengthen reliability of the FATCOD. The Pearson coefficient was determined to be 0.90 . The content validity index (CVI) of the FATCOD in 1988 was 1.00 with a computed interrater agreement of 0.98 . The CVI was repeated in 1998 with an inter-rater agreement of 1.00 .

Emotional intelligence was measured by the Mayer-SaloveyCaruso Emotional Intelligence Test (MSCEIT) which is an ability-based measure of emotional intelligence designed for adults ages 17 and over. ${ }^{[20]}$ The MSCEIT consists of 141 items designed to measure the four branches of emotional intelligence: perceiving emotions, using emotions to facilitate thought, understanding emotions, and managing emotions scores which comprise the total score. ${ }^{[21]}$ The total emotional intelligent score provides an overall index of the respondent's emotional intelligence. ${ }^{[22]}$ The split-half reliability coefficients for the four branches as reported in the technical manual range from $r=.80$ to .91 , and for the entire test $r=.91 .^{[23]}$ Validity has been addressed with the MSCEIT. Face validity is apparent in the tasks utilized by the instrument to assess emotional intelligence. ${ }^{[23]}$ Content validity is demonstrated by the MSCEIT's appropriate representation of the Four Branch Model. ${ }^{[23]}$ And finally, the finding in the 2003 study by Brackett and Mayer ${ }^{[21]}$ point to appropriate convergent, discriminant, and incremental validity of the MSCEIT. The above noted validity data offers empirical justification for the use of the MSCEIT. Prior to purchasing the MSCEIT, individuals lacking formal psychological training must be certified.

Self-reflection was measured by the Self Reflection and Insight Scale (SRIS) which is a 5 point Likert scale type questionnaire asking subjects the extent to which they agree or disagree with 20 statements. ${ }^{[24]}$ The responses to each question are scored on a scale of one to five with one equating to 
"strongly disagree" and five to "strongly agree". The statements relate to three domains of insight: recognition of the need for reflection, the process of engaging in reflection and the presence of insight. In a series of studies two separate factor analyses found the SRIS comprised two separate factors labeled Self-Reflection (SRIS-SR) and Insight (SRIS-IN). "Need for self-reflection" and "engagement in self-reflection" loaded on the same factor. Test-retest reliability over a 7-week period was .77 (SRIS-SR) and .78 (SRIS-IN). Validation of the SRIS was further confirmed through factor analysis with the conclusion that developing reflection and insight is especially important for medical professionals. ${ }^{[25]}$

In order to obtain data on the demographic variables, participants were asked to complete a demographic data sheet on-line. The demographic variables are age, gender, marital status, ethnicity, and religion.

\section{Statistical Analysis}

All data was analyzed using the PASW Statistics 18. Demographic variables that were nominal in nature (gender, marital status, race and religion) were analyzed using proportion (\%) and absolute number $(\mathrm{N})$ statistics. The demographic variable that yields interval data (age) was analyzed using measures of central tendency $(M)$, dispersion $(S D, R)$, proportion (\%), and absolute number $(\mathrm{N})$. Improvement scores were calculated for the intervention and control group by subtracting the mean post-scores from the mean pre-scores on communication skills, attitudes towards dying patients, emotional intelligence, and self-reflection and insight. Paired t-tests were used to compare differences in means between the improvement scores of the intervention and control groups on each variable. P-values less than .05 were considered statistically significant.

\section{Ethical Consideration}

Approval for the study was obtained from Fairfield University's Institutional Review Board. The researchers made an announcement in the Mental Health Course that participation in the study was voluntary and that any identifying information would be coded, no individual results would be shared, and no repercussions with regards to grades would occur.

\section{Procedure}

The researcher sent an electronic invitation to all the students inviting them to participate in the study. If students were willing and qualified to participate in the study after reading the invitation, they clicked on an embedded web link to access an established electronic survey. Once on the website, the participants were asked to electronically complete the demographic information, Frommelt Attitude Toward Care of the Dying Scale, and Self Reflection and Insight Scale (SRIS). Completion of this information took approximately 40 minutes. Participants completed the questions in one sitting and were permitted to skip questions.
In addition, participants were given a web link to access the Mayer-Salovey-Caruso Emotional Intelligence Test (MSCEIT). Participants were instructed to enter their first and last name. However, this information was later coded. The participants needed to complete the MSCEIT in one sitting and there was no imposed time limit. The MSCEIT typically requires between 30 to 45 minutes to complete.

The survey did not collect any additional information beyond the questions provided on the form. Students were allotted class time to complete the pre/post-intervention surveys. As an incentive, students were eligible to enter into a raffle to win a gift card if they completed the pre/post surveys. Process recordings were completed on the first and last day of clinical as part of the students' clinical experience.

The psychodrama sessions were a course requirement for the intervention group. All students in the intervention group were expected to participate and the time in psychodrama counted as part of their clinical experience. The voluntary aspect of this study was participating in the survey that assessed pre/post measures.

\section{Intervention}

During the semester, the students were part of a weekly psychodrama group that included 6 two-hour psychodrama sessions that counted towards the students' clinical hours. The typical psychodrama session was comprised of three parts: warm-up, enactment and sharing. In the warm-up part, techniques were used to develop group cohesion, focus the group on its task, or create a special atmosphere, orientation, or theme in a group. The enactment was characterized by playing the scenario created by group member. Group member were encouraged to continue and complete their actions through dramatizations, role-playing, and dramatic self-presentation. Both verbal and nonverbal communications were utilized. A number of scenes (memories, unfinished situations, inner experience, fantasies, dreams, risk-taking situations or simply unrehearsed expressions of mental states) were enacted. Sharing was a time for the auxiliaries to de-role, for the protagonist to reconnect with the group, and for the other group members to express their thoughts and feelings about what in their own lives was touched by the protagonist's or group story. In this study, in addition to the typical psychodrama session, the group leader discussed the theoretical aspects of the topic following the session. The psychodrama sessions included the following content:

Session 1: Introduction, self-knowledge

Session 2: Awareness attitudes regarding life and death as an individual and a professional

Session 3: Coping with patient responses

Session 4: Communication problems within groups; dealing with communication problems within families, working in teams

Session 5: Ethical/legal issues, and cross cultural challenges Session 6: Termination and goodbye. 
All groups were led by a Ph.D. psychiatric nurse trained in psychodrama and co-led by a doctoral nursing student. Each group consisted of 14 baccalaureate nursing students who were in clinical for the Mental Health Nursing. The nursing students attended the off campus clinical experience with a clinical instructor. Student returned to the university campus for the psychodrama sessions conducted by the Ph.D. psychiatric nurse trained in psychodrama who had no other responsibilities regarding the education of students. A certificate of completion was given to every student that completed the psychodrama sessions regardless of whether they complete the on-line surveys.

\section{Results}

Thirty-eight out of a possible 42 students participated in the intervention group ( $90 \%$ response rate) and 41 out of a possible 42 students participated in the control group (98\% response rate). Five characteristics were analyzed as demographic variable for this study (Table 1). Personal characteristics of the participants included age, gender, marital status, race, and religion. The age of the intervention sample ranged from 19-20 years old with a mean of 19.2. The age of the control sample ranged from 19-21 years old with a mean of 20.0. The majority of the participants in the intervention (89.5\%) and the control group $(95.1 \%)$ were female. All participants $(100 \%)$ in the intervention and control group were single. The majority of the nursing students in the intervention (94.7\%) and control $(87.8 \%)$ were Caucasian. And the majority of the participants in the intervention (73.7\%) and the control (80.5\%) were Catholic. Table 1 illustrates the demographic characteristics for the intervention and control group.
The improvement in communication skills, attitudes towards the care of the dying, self-reflection, and emotional intelligence of nursing students in the psychodrama group compared to those in the control group was analyzed by paired t-test analysis. There was a statistically significant difference in improved communication skills between the group receiving the 6-week psychodrama intervention and the control group $(\mathrm{t}=4.22, \mathrm{p}=.000)$. There was a statistically significant difference in improved attitudes between the group receiving the psychodrama intervention and the control group $(t=27.19$, $\mathrm{p}=.000$ ). And, there was a statistically significant difference in improved self-reflection between the group receiving the intervention and the control group ( $\mathrm{t}=5.60, \mathrm{p}=.000$ ).

However, there was no statistically significant difference in improved total emotional intelligence between the intervention and control group ( $\mathrm{t}=.45, \mathrm{p}=.657$ ). Nor was there a statistically significant difference in improved branch 1 of emotional intelligence (perceiving emotional intelligence) $(t=1.48, p=.143)$ between the intervention and control group. In addition, there was no statistically significant difference in improved branch 2 of emotional intelligence (using emotions to facilitate thoughts) ( $t=-.51, p=.613$ ) between the intervention and control group. Furthermore, there was no statistically significant difference in improved branch 3 of emotional intelligence (understanding emotions) ( $t=-1.53, p=.129)$ between the intervention and control group. And finally, there was no statistically significant difference in improved branch 4 of emotional intelligence (managing emotions) $(t=.67, p=.505)$ between the intervention and control group. Of note, there was no statistical difference in the tasks of emotional intelligence except for one task (understanding emotions-blends task) ( $t=-2.03$, $\mathrm{p}=.046$ ). These results are displayed in Table 2 .

\begin{tabular}{lcc} 
Table 1. Characteristics of the intervention $(\mathbf{n}=\mathbf{3 8})$ and control $(\mathbf{n}=\mathbf{4 1})$ group participants \\
\hline Characteristic & Intervention group $\mathbf{n}(\%)$ & Control group $\mathbf{n}(\%)$ \\
\hline $\begin{array}{l}\text { Gender } \\
\text { Female }\end{array}$ & $34(89.5)$ & $39(95.1)$ \\
$\quad$ Male & $4(10.5)$ & $2(4.9)$ \\
Marital status & & \\
$\quad$ Single & $38(100)$ & $41(100)$ \\
Race & & \\
Caucasian & $36(94.7)$ & $36(87.8)$ \\
Black/African American & $1(2.6)$ & $1(2.4)$ \\
Hispanic/Latino & $1(2.6)$ & $2(4.9)$ \\
Other & $0(0)$ & $2(4.9)$ \\
Religion & & \\
Catholic & $28(73.7)$ & $33(80.5)$ \\
Protestant & $1(2.6)$ & $0(0)$ \\
Christian & $5(13.2)$ & $2(4.9)$ \\
Non-denominational & $1(2.6)$ & $0(0)$ \\
None & $3(7.9)$ & $2(4.9)$ \\
Other & $0(0)$ & $3(7.3)$ \\
\end{tabular}


Table 2. Paired t-test results comparing differences in means between the improvement scores of the intervention ( $n=38$ ) and control $(n=41)$ groups on each variable

\begin{tabular}{|c|c|c|c|c|c|c|c|}
\hline \multirow[b]{2}{*}{ Variable } & \multicolumn{2}{|c|}{$\begin{array}{l}\text { Intervention } \\
\qquad(n=38)\end{array}$} & \multicolumn{2}{|c|}{$\begin{array}{l}\text { Control } \\
(n=4 I)\end{array}$} & \multirow[b]{2}{*}{$\mathbf{t}$} & \multirow[b]{2}{*}{ df } & \multirow[b]{2}{*}{ sig. (2 tailed) } \\
\hline & M & SD & $\mathbf{M}$ & SD & & & \\
\hline Communication skills & 9.29 & 5.36 & 3.38 & 6.87 & 4.22 & 76 & .000 \\
\hline Attitudes towards the care of the dying & 68.34 & 13.95 & .37 & 7.57 & 27.19 & 77 & .000 \\
\hline Self-reflection and insight & 23.92 & 20.90 & 3.98 & 8.81 & 5.60 & 77 & .000 \\
\hline Branch 1 of emotional intelligence (perceiving emotions) & 1.02 & 10.17 & -3.47 & 15.96 & 1.48 & 77 & .143 \\
\hline Branch 2 of emotional intelligence (using emotions to facilitate thoughts) & -.75 & 9.76 & .33 & 9.19 & -.51 & 77 & 613 \\
\hline Branch 3 of emotional intelligence (understanding emotions) & -.84 & 9.01 & 2.36 & 9.47 & -1.53 & 77 & .129 \\
\hline Branch 4 of emotional intelligence (managing emotions) & 1.13 & 9.92 & -.39 & 10.25 & .67 & 77 & .505 \\
\hline Task score of understanding emotions-blends task & -1.63 & 10.62 & 3.22 & 10.62 & -2.03 & 77 & .046 \\
\hline
\end{tabular}

\section{Discussion}

\section{Discussion of the Response Rate}

Thirty eight students ( $90 \%$ response rate) of the intervention group and forty one students (98\%) of the control group responded to the pre and post surveys. This response rate exceeds the response rate supported by Dillman ${ }^{[26]}$ who report that the average response for email surveys with two contacts is 41 percent. This is most likely related to the fact that the faculty allowed class time for the completion of the questionnaires.

\section{Discussion of the Sample}

The 79 nursing students (38 in the control group and 41 in the intervention group) were mostly single, Caucasian, Catholic, females between the ages of 19-21 years old. All students were in the traditional undergraduate program. The sample was a relatively small, homogeneous group making it difficult to generalize beyond the undergraduate population.

\section{Discussion of the Results}

Does an end-of-life psychodrama intervention influence nursing students' communication skills? Results of this study offer preliminary evidence that nursing students and subsequently, their patients, can benefit from receiving psychodrama education to improve end-of-life communication skills. The significant difference in average improved scores in communication skills in this study supports the previous study by Ozcan, Bilgin and Eracar ${ }^{[17]}$ in which psychodrama helped to improve empathy. Empathy is one of many effective communication skills that is especially relevant during end-of-life care. A typical mental health class tends to address communication skills by focusing on an understanding of the patient. Whereas the psychodrama strategy implemented in the intervention group considers the student nurse's experiences as well as the patient's in an effort to improve communication. Hence, adding psychodrama to the frequently used method of focusing on specific communication skills may be more effective in reaching desired outcomes. Better communication skills mean nurses will be better able to deliver compassionate care, patient and family education, and facilitate challenging situations that arise in practice.

Does an end-of-life psychodrama intervention influence nursing students' attitudes towards dying patients? The results of this study offer evidence that nursing students can benefit from receiving psychodrama education to improve their attitudes towards the care of the dying. Although there were no previous studies found that examined attitudes towards the dying after a psychodrama intervention, the findings are logical given that the self-reflection and insight scores increased which in turn may help to promote improved attitudes toward the experience.

Does an end-of-life psychodrama intervention influence nursing students' emotional intelligence? The results of this study indicate that psychodrama did not help to improve total and emotional intelligence scores. However, there was a difference in average improved scores in one of the tasks scores of emotional intelligence for the intervention and control group. This finding supports that although such an intervention might not influence overall emotional intelligence it may help to enhance some of the emotional intelligence skills. This substantiates the study by Ozcan et al. ${ }^{[17]}$ that found that psychodrama helped to improve empathy in nursing students (empathy being a closely related concept to emotional intelligence). This is one of the first studies to examine if an intervention such as psychodrama can improve emotional intelligence in nursing students. More studies need to be completed to see if other interventions can help to enhance emotional intelligence skills.

Does an end-of-life psychodrama intervention influence nursing students' self-reflection and insight? The results of the study also provide preliminary evidence that nursing students can benefit from receiving psychodrama education to im- 
prove their self-reflection and insight. This supports the previous study by Oflaz et al. ${ }^{[6]}$ in which psychodrama helped to improve self-awareness in nursing students. In addition, it supports the findings of the study by Giusti et al. ${ }^{[16]}$ which found that psychodrama helped cancer patients achieve insights. Psychodrama appears to be a useful educational tool to promote self-awareness and insight. This strategy assists student nurses to understand themselves as well as understand the perspectives of their patients at the end-of-life.

\section{Implications for Nursing Education and Practice}

The results have important implications for nursing education. Given that the end-of-life psychodrama had a positive influence on nursing student outcomes (i.e. communication skills, attitudes towards care of those at the end-of-life, self-reflection and emotional intelligence), then psychodrama ought to be considered as a viable teaching strategy in nursing curriculum. These outcomes are not enhanced by merely reading content regarding end-of-life. Rather, more active engaged teaching methods such as psychodrama have added value. Psychodrama can foster improved attitudes, self-awareness, and enhanced emotional intelligence skills that can ultimately lead to improved communication skills. Those communication skills can be practiced during psychodrama until they are mastered and become routine for the nursing student. Inclusion of psychodrama is feasible for nursing programs. Faculty can be educated and certified in psychodrama. Once properly trained, faculty can incorporate the psychodrama learning strategy as part of the students' clinical/laboratory/simulation/ classroom hours.

In addition to nursing education, the results have implications for nursing practice. End-of-life psychodrama for health professionals can be used in hospice and oncology healthcare settings to improve communication skills, attitudes, self-reflection and emotional intelligence skills in all types of nursing personnel from bedside staff to leaders. However, it is reasonable to think that psychodrama would be beneficial in other nursing specialties (not just those affected by end-of-life issues) given that these outcomes are universal and vital in all healthcare settings.

\section{Selected Limitations of the Study}

Given that the study utilized a small convenience sample, the subjects may not be representative of all nursing students. Therefore, the results cannot be generalized. In addition, the setting (a private Jesuit university in the United States) is not representative of the setting of all nursing programs and may have an effect on the self-reflection and insight score as reflection is an integral part of the Ignation pedagogy used in Jesuit universities. Another potential limitation was that the psychodramatist was a visiting professor from Turkey; and English was a second language for her. Finally, the self-report instrument used to measure attitudes towards care of the dy- ing and self-reflection and insight can be susceptible to potential bias.

\section{Future Research}

Replication of this study across cultures is suggested. Additional research exploring strategies to promote effective nursing communication during the end-of-life are necessary. Further studies are also needed to examine interventions that might enhance emotional intelligence.

\section{Conclusion}

Psychodrama is a participatory action method that uses spontaneous dramatization, role playing, and dramatic self-presentation to enhance and gain insight and can be utilized across multiple contexts. ${ }^{[27,28]}$ Since communication skills at the endof-life have been identified as lacking, it is critical that nursing students receive extensive training to foster these skills and ensure excellent end-of-life care as it will help foster the healing capacities of individuals, groups and families. Improving students' ability to communicate will ultimately result in better client outcomes and higher quality of care across multiple contexts. The landscape of healthcare is changing with the increase in the elderly population in tandem with increased numbers of culturally diverse patients who seek care. The innovative educational approach utilized in this study provides a model that can be adapted across cultures to help nursing students assist the healing and recovery process for individuals at the end-of-life and their families.

\section{Acknowledgements}

This work was supported by the Marion Peckham Egan School of Nursing \& Health Studies and Summer Research Stipend at Fairfield University, Fairfield, CT USA.

Conflict of interest: There are no relevant conflicts of interest to disclose.

\section{Peer-review: Externally peer-reviewed.}

Authorship contributions: Concept - A.A.Ö., K.W.; Design A.M.B., A.A.Ö., K.W.; Supervision - A.M.B., A.A.Ö., K.W.; Fundings - A.M.B., K.W.; Materials - A.M.B., A.A.Ö.; Data collection \&/or processing - A.M.B., A.A.Ö.; Analysis and/or interpretation - A.M.B., A.A.Ö., K.W.; Literature search - A.M.B., A.A.Ö., K.W.; Writing A.M.B., A.A.Ö., K.W.; Critical review - A.M.B., A.A.Ö., K.W.

\section{References}

1. Gillett K, O'Neill B, Bloomfield JG. Factors influencing the development of end-of-life communication skills: A focus group study of nursing and medical students. Nurse Educ Today 2016;36:395-400.

2. Barnes S, Gardiner C, Gott M, Payne S, Chady B, Small N, et al. Enhancing patient-professional communication about endof-life issues in life-limiting conditions: a critical review of the 
literature. J Pain Symptom Manage 2012;44:866-79.

3. Grant MS, Jenkins LS. Communication education for pre-licensure nursing students: literature review 2002-2013. Nurse Educ Today 2014;34:1375-81.

4. Baile WF, Walters R. Applying sociodramatic methods in teaching transition to palliative care. J Pain Symptom Manage 2013;45:606-19.

5. Sahebalzamani M, Farahani $H$, Moeini F, Rashvand F. The effect of emotional intelligence training via psychodrama method on emotional reactions of MS patients. Journal of Applied Environmental and Biological Sciences 2014;4:63-7.

6. Oflaz F, Meriç M, Yuksel Ç, Ozcan CT. Psychodrama: an innovative way of improving self-awareness of nurses. J Psychiatr Ment Health Nurs 2011;18:569-75.

7. Mayer JD, Salovey P. What is emotional intelligence? In: Salovey P, Sluyter D, editors. Emotional Development and Emotional Intelligence: Implications for Educators. New York: Basic Books; 1997. p. 3-31.

8. Erigüç $\mathrm{G}$, Kose $\mathrm{S}$. Evaluation of emotional intelligence and communication skills of health care manager candidates: $A$ structural equation modeling. International Journal of Business and Social Science 2013;4:115-23.

9. Erigüç $G$, Eriş $H$, Kabalcıoğlu F. Emotional Intelligence and communication skills of nursing students: Example of Harran University School of Health. International Online Journal of Educational Sciences 2014;6:398-412.

10. Martos MP, Lopez-Zafra E, Pulido-Martos M, Augusto JM. Are emotional intelligent workers also more empathic? Scand J Psychol 2013;54:407-14.

11. Nikbakhsh R, Alam SB, Monazami M. The relationship between emotional intelligence, communication skills and stress among Iranian premier league referees. Annals of Biological Research 2013;4:196-203.

12. Beauvais AM, Brady N, O'Shea ER, Griffin MT. Emotional intelligence and nursing performance among nursing students. Nurse Educ Today 2011;31:396-401.

13. Beauvais A, Stewart JG, DeNisco S. Emotional intelligence and spiritual well-being: implications for spiritual care. J Christ Nurs 2014;31:166-71.

14. Beauvais AM, Stewart JG, DeNisco S, Beauvais JE. Factors related to academic success among nursing students: a descriptive correlational research study. Nurse Educ Today 2014;34:918-23.

15. Özbap AA, Tel H. The effect of a psychological empower- ment program based on psychodrama on empowerment perception and burnout levels in oncology nurses: Psychological empowerment in oncology nurses. Palliat Support Care 2016;14:393-401.

16. Menichetti J, Giusti L, Fossati I, Vegni E. Adjustment to cancer: exploring patients' experiences of participating in a psychodramatic group intervention. Eur J Cancer Care (Engl) 2016;25:903-15.

17. Ozcan NK, Bilgin H, Eracar N. The use of expressive methods for developing empathic skills. Issues Ment Health Nurs 2011;32:131-6.

18. Kissane DW, Bylund CL, Banerjee SC, Bialer PA, Levin TT, Maloney EK, et al. Communication skills training for oncology professionals. J Clin Oncol 2012;30:1242-7.

19. Frommelt $\mathrm{KH}$. The effects of death education on nurses' attitudes toward caring for terminally ill persons and their families. Am J Hosp Palliat Care 1991;8:37-43.

20. Mayer JD, Salovey P, Caruso DR, Sitarenios G. Measuring emotional intelligence with the MSCEIT V2.0. Emotion 2003;3:97105.

21. Mayer JD, Salovey P, Caruso D. Mayer-Salovey-Caruso Emotional Intelligence Test (MSCEIT) user's manual. Toronto, Multi-Health Systems; 2002.

22. Multi-Health Systems. MHS: 2006 Catalog. Toronto, Canada; 2006.

23. Mayer JD, Salovey P, Caruso D. MSCEIT technical manual. Toronto, Canada: Multi-Health Systems; 2000.

24. Grant AM, Franklin J, Langford P. The Self-reflection and Insight Scale: a new measure of private self-consciousness. Social Behavior and Personality An International Journal 2002;30:821-36.

25. Roberts C, Stark P. Readiness for self-directed change in professional behaviours: Factorial validation of the Self-reflection and Insight Scale. Medical Education 2008;42:1054-1063.

26. Dillman DA. Mail and internet surveys: The tailored design method. 2nd ed. USA: Hoboken, NJ: John Wiley \& Sons Inc; 2007.

27. Gershoni J. Psychodrama in the 21 st Century Clinical and Educational Applications. Springer Publishing Company; 2013.

28. Hollander, CE. A process for psychodrama training: The Hollander Psychodrama Curve. International Journal of Action Methods: Psychodrama, Skill Training, and Role Playing 2002;54:147-57. 